\title{
Continuing professional development: reflective practice
}

\section{Ekanayake $\mathbf{C}^{\mathbf{l}}$}

1Consultant Obstetrician and Gynaecologist and Senior Lecturer, Department of Clinical Sciences, Faculty of Medicine, General Sir John Kotelawala Defense University.

\section{Corresponding Author - Dr Chanil Ekanayake}

Email-cdekanayake2000@yahoo.com

A quote by Confucius states, "by three methods we may learn wisdom: by experience which is the bitterest, by imitation which is the easiest and by reflection which is the noblest".

Reflection must not be limited to just clinical practice and should involve data fiom audits, compliments as well as complaints, workbased assessments and feedback from staff and patients.

Reflective practice is a mandatory component that should be included in the portfolio of postgraduate trainees prior to board certification in Sri Lanka. A reflective practice scenario is included as an example. Personal details have been excluded for purposes of confidentiality. Consent was obtained to use images for purposes of learning.

\section{Delayed diagnosis of an endometrial carcinoma}

A 63 year old lady from a rural setting presented with a blood stained vaginal discharge for one year. She had presented to the local hospital at the onset of her problem and had undergone an examination under anaesthesia and dilatation and curettage $(E U A+D \& C)$. Although she had attended the gynaecology clinic the histology had not been available and this prompted her to default follow up. Due to persuasion by her daughter she attended a gynaecology clinic in another province four hours from her hometown.

She had reached menopause at 54 years and had not used any hormone replacement therapy (HRT). She could not exactly remember when she attained menarche. She did not have a history of oligomenorthoea or polycystic ovarian syndrome and had not undergone any surgery. There were no medical co-morbidities. She was a mother of one child. There was no family history of malignancy. She had never had a cervical smear. Her BMI was $35 \mathrm{~kg} / \mathrm{m} 2$. Cardiovascular and respiratory systems were clinically normal. Abdominal examination did not reveal any masses. Speculum examination revealed a healthy cervix and blood stained discharge through the cervical OS. Uterus felt bulky with no adnexal masses. A transvaginal ultrasound scan (TVS) showed pyometron with an endometrial thickness (ET) of $34 \mathrm{~mm}$. Papillary structures were seen within the uterine cavity. A pipelle aspiration was done in the clinic itself. Histology revealed a benign polyp with a suggestion to repeat if suspicious for endometrial cancer.

This patient was at a very high risk for endometrial cancer due to the presence of a blood stained discharge, TVS findings of a very high ET and papillary structures within the uterine cavity, low parity and obesity. Therefore a decision was taken not to repeat a pipelle aspiration or an evaluation under anaesthesia with dilatation and curettage (EUA+D\&C), but to go ahead with a $\mathrm{TAH}+\mathrm{BSO}$ (total abdominal hysterectomy and bilateral salphingo-oophorectomy) with a view to do a pelvic lymphadenectomy (PND) if needed.

A midline incision was made and the peritoneal cavity entered into in an uneventful manner. A bulky fibroid uterus noted and a $\mathrm{TAH}+\mathrm{BSO}$ was done. The specimen was cut open in the op- 
erating theatre which showed $>50 \%$ myometrial involvement with possible cervical involvement. Therefore a pelvic lymphadenectomy was done. As there were papillary structures within the uterine cavity the possibility of a serous papillary endometrial cancer was entertained which prompted an infracolic omentectomy.

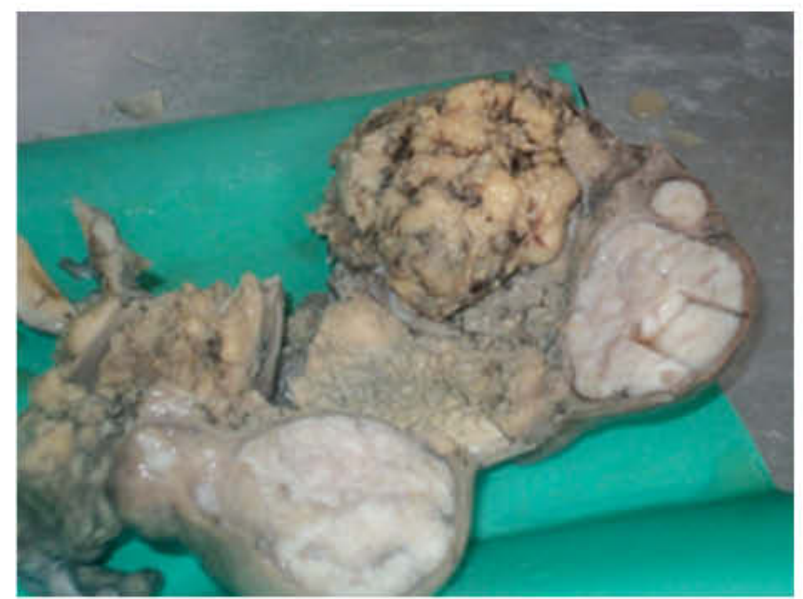

Figure 01.

Macroscopic apperance of the uterus

The uterine cavity exhibited a tumour $7.5 \mathrm{X} 6 \mathrm{X}$ $4 \mathrm{~cm}$ in size (Figure 01). The tumour was seen to extend into the cervix with $>90 \%$ of the myometrial involvement at the maximum point of invasion (Figure 02). Multiple well defined intramural nodules were seen, ranging in size from $0.8-3.8 \mathrm{~cm}$.
A malignant tumour in the endometrium composed of complex acinar and papillary structures was seen with solid areas $<5 \%$ (Grade 1 ). The cells showed mild to moderate nuclear pleomorphism (nuclear grade 1-2) [Figure 03]. At the maximum point of invasion the tumour was only $0.3 \mathrm{~mm}$ from the serosa. Tumour involvement was seen in the cervical stroma [Figure 04].

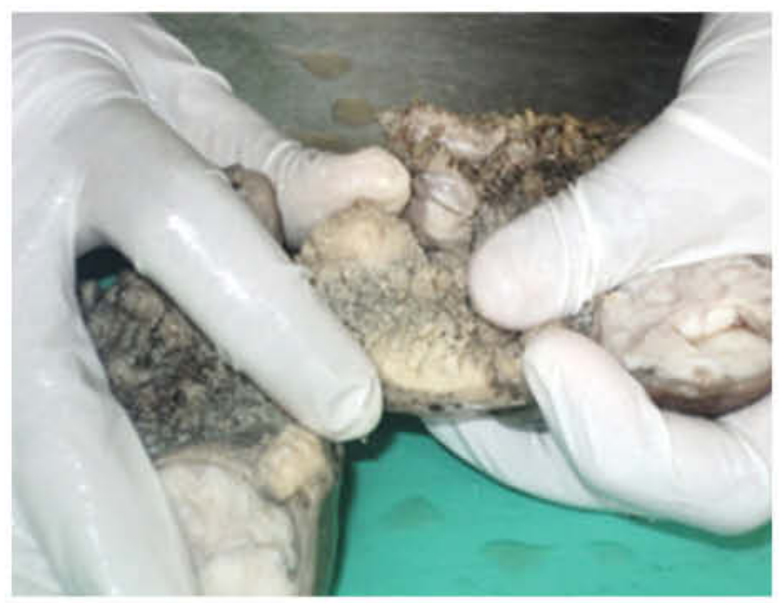

Figure 02.

Cervical Involvement

Lympho-vascular invasion was present. Both ovaries showed metastatic tumour deposits. Regional lymph nodes were free of tumour deposits. TNM staging was pT3aN0Mx. FIGO Stage IIIA. Patient was subsequently referred to the National Cancer Institute for adjuvant radiotherapy.

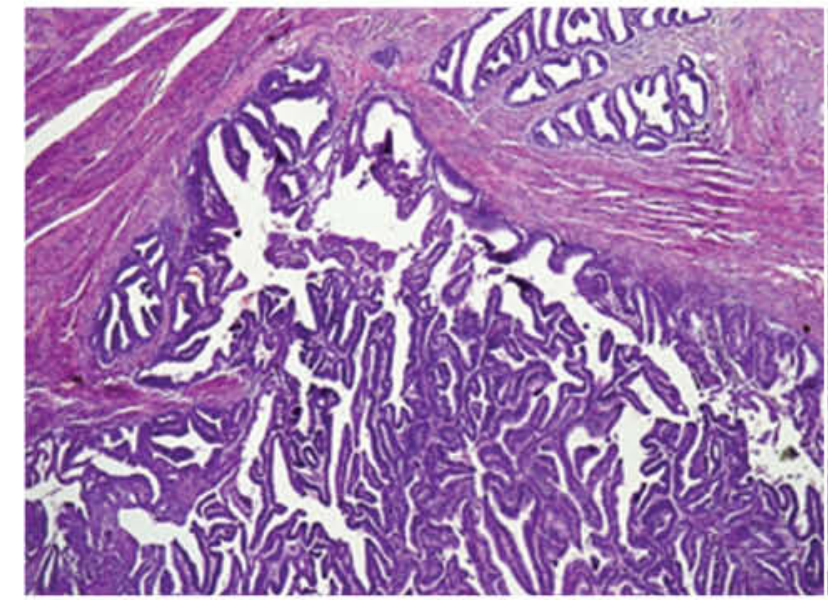

Figure 03.

Endometrial cancer with complex acinar and papillary structures

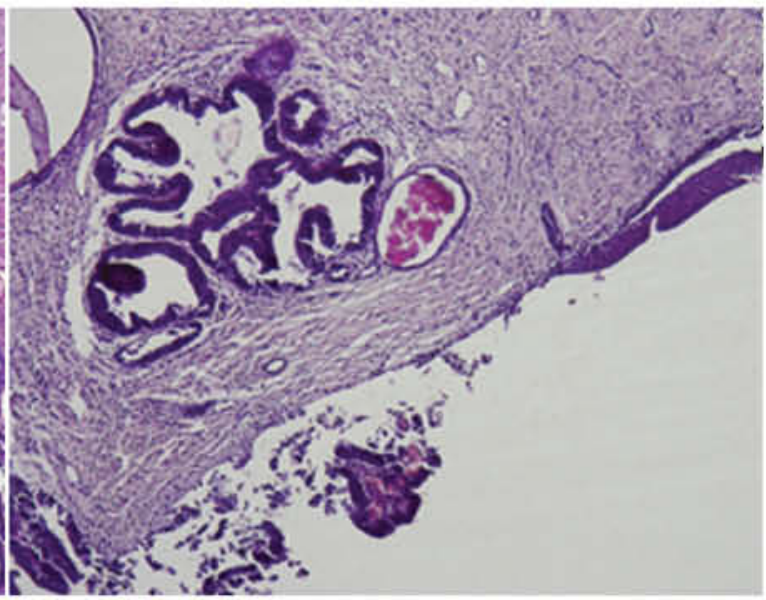

Figure 04.

Tumour invasion of the cervical stroma 
Issues identified

1. Delayed diagnosis of endometrial cancer

2. What is the best method of endometrial sampling?

3. Preoperative staging investigations

4. Decision for total abdominal hysterectomy, bilateral salphingo-oophorectomy and pelvic lymphadenectomy

5. Whether pelvic lymphadenectomy (PND)?

\section{Delayed diagnosis of endometrial cancer}

A diagnosis of stage III A endometrial cancer was made according to the revised FIGO criteria for staging endometrial carcinoma ${ }^{1}$. However, it would seem obvious that this patient was at a very high risk for endometrial carcinoma (as explained above) right from the outset. Therefore the histology result should have been followed up more intensely and a repeat procedure done if the specimen was not traceable. Perhaps the reason for this is because the local hospital was a busy centre with no postgraduates and only two Gynaecologists serving a large population. Thus, the histology was probably followed up by junior doctors who may not have done a proper assessment of the risk of malignancy.

A centralized database within the ministry of health would have alleviated the problem but this is a far cry from reality in the current context. Therefore measures should have been taken to contact the relevant unit. However, this issue was conveyed to the local gynaecology unit.

\section{What is the best method of endometrial sampling?}

Current evidence suggests that hysteroscopy is by far the gold standard to exclude endometrial cancer as a positive hysteroscopy has a likelihood ratio (LR) of 62.8 (95\% CI 52.8-74.6), while a negative hysteroscopy has a LR of 0.15 (95\% CI $0.13-0.18)^{2}$.

Although an EUA+D\&C is an outdated method of endometrial sampling, it is still the method of choice in peripheral centres in Sri Lanka as hysteroscopy and pipelle aspirators are not available due to financial constraints ${ }^{3}$. A rethink of this policy should be made as pipelle aspiration is $99.6 \%$ sensitive in detecting endometrial cancer ${ }^{2}$. Furthermore, as it is a less invasive out-patient procedure as opposed to a EUA+D\&C, it is probably more cost-effective as well. The other advantage is that if a diagnosis of cancer is made it effectively negates the need of the patient being anaesthetised to achieve a histological diagnosis prior to definitive surgery. In this regard it has an advantage over hysteroscopy, the current gold standard method. One may argue that out-patient hysteroscopy would still have an advantage, but it is still not a reality in most centres worldwide.

Insufficient sampling often puts the clinician in a dilemma. If the ET is $>4 \mathrm{~mm}$, an insufficient sample should not be taken lightly as there is a possibility of endometrial cancer or atypical hyperpla$\mathrm{sia}^{3}$. Thus, measures should be taken to resample the endometrium if the ET $>4 \mathrm{~mm}$. Clinicians can reassure patients with an insufficient sample, provided that there is hysteroscopic evidence of endometrial atrophy or if the ET is $<4 \mathrm{~mm}^{3}$. Even still, there is a need to reinvestigate if there is a recurrence of symptoms as a small polyp in an atrophic endometrium may be missed if the sampling is inadequate ${ }^{3}$.

\section{Preoperative staging investigations}

FIGO staging in endometrial cancer is essentially surgical and involves a preoperative chest X-ray, $\mathrm{TAH}+\mathrm{BSO}$ and pelvic and para-aortic lymphadenectomy ${ }^{1}$. Despite this it is better to embark on surgery knowing the extent of the disease as it allows better planning of surgery. It is in this light that preoperative investigations gain relevance. Computed tomography (CT) abdomen and pelvis is the most useful in defining extrauterine and extra pelvic spread ${ }^{1}$. Magnetic resonance imaging (MRI) is the best method of defining myometrial spread and cervical involvement. However cost constraints make these a difficult proposition in the government sector in Sri Lanka. Therefore our practice is to cut open the uterine specimen 
following hysterectomy and assess the degree of myometrial involvement. Lymph node involvement is assessed on naked eye appearance.

4. Decision for total abdominal hysterectomy, bilateral salphingo-oophorectomy and pelvic lymphadenectomy

As this patient had two inconclusive attempts at a diagnosis in the backdrop of multiple risk factors a decision was taken to proceed with definite surgery. It was a brave decision, but a correct and decisive decision which had a positive impact on the course of her illness.

\section{To do or not to do pelvic lymphadenectomy (PND)?}

There is raging debate on the role of pelvic lymphadenectomy in endometrial cancer. North American practice justifies the need for a full surgical staging involving PND as it triages node negative women to surveillance only, while node positive women are offered adjuvant treatment.
Thus avoiding over and undertreatment ${ }^{4}$. The UK practice is to defer PND as they think it does not confer a therapeutic benefit nor help in triaging cases. They argue that triaging can effectively be achieved using the histological specimen alone and that the therapeutic benefit of adjuvant radiotherapy is seen in both women who have undergone and those who have not undergone PND. They also state that the added morbidity of PND, particularly in the elderly and obese patients is not justifiable 5 .

Our practice is to do PND due to the following reasons;

- It allows a full surgical staging to be done

- Limited facilities to provide adjuvant radiotherapy in a timely manner

- High degree of morbidity from radiotherapy as most patients do not receive lesion targeted/mapped therapy using linear accelerators

A summary of the management of early stage endometrial cancer and the justifications for it is shown (Figure 05).

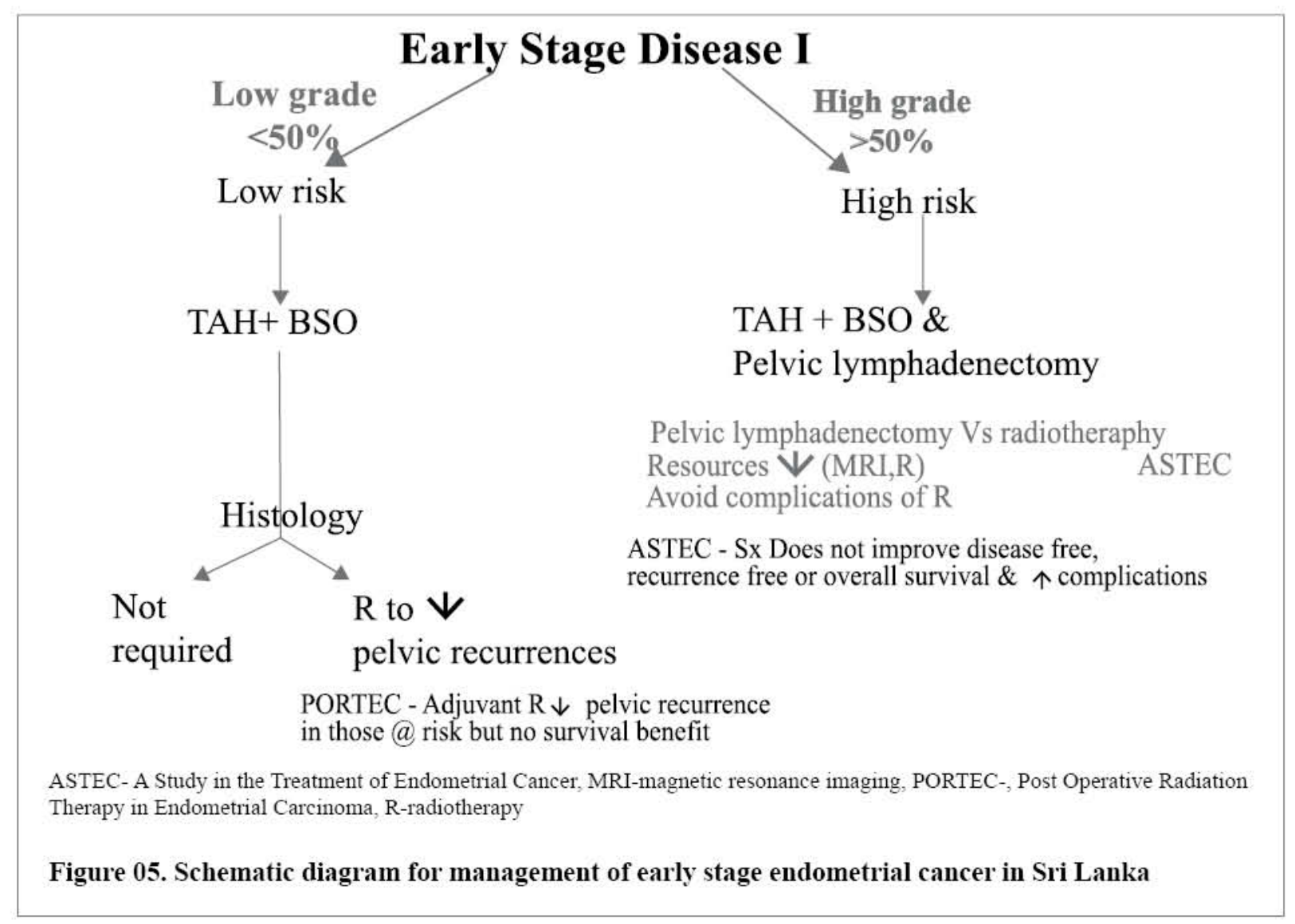


The following are some important points in managing patients at risk of endometrial cancer

\section{- Presence of fluid in the uterine cavity}

Although the presence of fluid in the uterine cavity is a good marker for endometrial pathology, if the ET is $<4 \mathrm{~mm}$ it need not be investigated unless there are other concerns ${ }^{3}$.

- Thickened endometrium in asymptomatic postmenopausal women: should it be investigated?

This problem is encountered when postmenopausal women undergo pelvic scans for reasons other than vaginal bleeding. TVS has a high sensitivity as a screening test for endometrial cancer ${ }^{3}$. There is no universal cut off for this group of patients. Endometrial polyps are the commonest pathology found in these cases. Although the risk of malignancy appears to be associated with polyp diameter, a cut off value is not known.

\section{- Tamoxifen and PMB}

Tamoxifen is selective estrogen receptor modulator that has a weak estrogenic effect on the endometrium. It is used extensively in breast cancer treatment. Treatment beyond 5 years raises the risk of endometrial cancer by four fold ${ }^{6}$. However periodic investigations are not cost-effective and PMB should be the trigger when investigating women on tamoxifen ${ }^{3}$.

\section{- Unscheduled bleeding in patients on HRT}

Bleeding is considered abnormal in women on sequential regimens if ${ }^{6}$

- It is heavy or prolonged at the end of, or after, the progestogen phase

- It occurs at any time (breakthrough bleeding)

Bleeding is considered abnormal in women on continuous combined regimens if (Scottish Intercollegiate Guidelines Network)

- It occurs after the first 6 months of treatment

- It occurs after the amenorrhoea has been established

\section{Conclusion}

Recurrent or persistent postmenopausal bleeding/ discharge should be considered a sentinel event as there is a high risk of malignancy. If a procedure is done appropriate follow-up is mandatory, especially histology. In the current context, local measures should be in place for adequate follow up in each hospital. Continuing medical education in the form of risk management meetings should be done for trainees and para medical staff of the unit.

\section{$\underline{\text { Acknowledgements }}$}

Prof. PS Wijesinghe, my supervisor and mentor should be praised for guiding me through this case during my senior registrar year. I would also like to acknowledge Dr. Kanishka Karunaratne (Consultant Gynaecological Oncosurgeon) for his specialist input during the final phase of manuscript preparation. The effort of Dr. Yohan Sachintha Silva, Senior Registrar should also be accredited.

\section{$\underline{\text { References }}$}

1) Edey K, Murdoch J. FIGO staging in vulval and endometrial cancer. The Obstetrician \& Gynaecologist 2010; 12:245-249

2) Clark T, Voit D, Gupta J, Song F, Khan K. Accuracy of hysteroscopy in the diagnosis of endometrial cancer and hyperplasia: a systematic quantitative review. JAMA 2002; 288:1610-1621

3) Bakour SH, Timmermans A, Mol BW, Khan KS. Management of women with postmenopausal bleeding: evidence-based review. The Obstetrician \& Gynaecologist 2012; 14:243249

4) Mariani A, Dowdy SC, Podratz KC. The role of pelvic and para-aortic lymph node dissection in the surgical treatment of endometrial cancer: a view from the USA. The Obstetrician \& Gynaecologist 2009; 11:199-204 
5) Holland CM. The role of pelvic and para-aortic lymph node dissection in the surgical treatment of endometrial cancer: a view from the UK. The Obstetrician \& Gynaecologist 2009; 11:205-209

6) Scottish Intercollegiate Guidelines Network (2002). Investigation of Postmenopausal Bleeding Section 5:Interpretation of Transvaginal Ultrasound (TVUS). Edinburgh: SIGN; [http://www.sign.ac.uk] 